Research Article

\title{
Graphical Analysis of Covering and Paired Domination in the Environment of Neutrosophic Information
}

\author{
Sami Ullah Khan, ${ }^{1}$ Abdul Nasir, ${ }^{1}$ Naeem Jan $\mathbb{D}^{1},{ }^{1}$ and Zhen-Hua Ma $\mathbb{D}^{2}$ \\ ${ }^{1}$ Department of Mathematics and Statistics, Institute of Numerical Sciences, Gomal University, Dera Ismail Khan, \\ KPK 29050, Pakistan \\ ${ }^{2}$ Department of Mathematics and Physics, Hebei University of Architecture, Zhangjiakou 075024, China
}

Correspondence should be addressed to Zhen-Hua Ma; mazhenghua_1981@163.com

Received 4 February 2021; Revised 19 February 2021; Accepted 2 March 2021; Published 19 April 2021

Academic Editor: G. Muhiuddin

Copyright (c) 2021 Sami Ullah Khan et al. This is an open access article distributed under the Creative Commons Attribution License, which permits unrestricted use, distribution, and reproduction in any medium, provided the original work is properly cited.

\begin{abstract}
Neutrosophic graph (NG) is a powerful tool in graph theory, which is capable of modeling many real-life problems with uncertainty due to unclear, varying, and indeterminate information. Meanwhile, the fuzzy graphs (FGs) and intuitionistic fuzzy graphs (IFGs) may not handle these problems as efficiently as NGs. It is difficult to model uncertainty due to imprecise information and vagueness in real-world scenarios. Many real-life optimization problems are modeled and solved using the wellknown fuzzy graph theory. The concepts of covering, matching, and paired domination play a major role in theoretical and applied neutrosophic environments of graph theory. Henceforth, the current study covers this void by introducing the notions of covering, matching, and paired domination in single-valued neutrosophic graph (SVNG) using the strong edges. Also, many attention-grabbing properties of these concepts are studied. Moreover, the strong covering number, strong matching number, and the strong paired domination number of complete SVNG, complete single-valued neutrosophic cycle (SVNC), and complete bipartite SVNG are worked out along with their fascinating properties.
\end{abstract}

\section{Introduction}

There are several techniques and methods to model real-life events. But, in practicality, the information is sometimes uncertain, unclear, varying, and indeterminate, which is difficult to model using usual methods. Different theories are proposed for modeling uncertainty. Smarandache [1] gave a novel concept of expressing and solving uncertainty. $\mathrm{He}$ initiated the neutrosophic sets (NSs), which are the extensions of fuzzy sets (FSs) and intuitionistic fuzzy sets (IFSs). FSs proposed by Zadeh [2] and their generalizations are used to model the uncertainty. FSs involve the membership grade for each object whose value lies in the unit interval $[0,1]$. The concept of IFSs was proposed and introduced by Atanassov in 1983 [3-5]. These are the generalization of FSs. The extension in IFSs is that in these sets the members of IFSs are assigned the membership grade as well as the nonmembership grade, provided that the sum of both grades lies in the unit interval $[0,1]$. Atanassov also discussed the types, properties, and applications of IFSs. But these concepts are unable to model many real-life problems, so Smarandache [6] devised the notion of NSs that can deal with such problems. In NS, the object carries three independent grades: membership, abstinence, and nonmembership. Each of these grades lies in nonstandard unit interval $]^{-} 0,1^{+}[$and their sum lies in nonstandard interval $]^{-} 0,3^{+}$. Later the concept of single-valued neutrosophic set (SVNS) was introduced by Wang et al. [7], which is the subclass of NSs. This is even more practical and applies to a wide range of problems.

Graph theory is one of the major branches of mathematics and combinatorics. It has many applications in numerous fields such as computer science, networking, geometry, algebra, set theory, economics, medicine, engineering, and chemistry. Hence, it has a wide range of applications in real world. Cantor presented the notion of crisp graphs, which uses the concepts of classical set theory. These graphs cannot model the problems associated with 
uncertainty because, in crisp graph, there are only two possibilities for a vertex and an edge, that is, whether it belongs to the graph or does not. In other words, it only conveys that the objects are related or not but does not indicate the strength and weakness of the relationship. So, an extension of crisp graphs is a fuzzy graph (FG), which was introduced by Rosenfeld in 1975 [8]. The idea of fuzziness in graph theory was given by Kauffman [9] in 1973 by using the fuzzy relations. Rosenfeld [8] further introduced the concepts related to FG such as connectedness, paths, cycles, trees, and bridges. Moreover, he also described some of the properties of FGs. Later on, the work of Rosenfeld was extended by many researchers who discussed its types, properties, and applications in real life, such as regular fuzzy graphs [10], fuzzy tolerance graphs [11], bipolar fuzzy graphs [12-14], interval-valued fuzzy graphs, balanced intervalvalued fuzzy graphs $[15,16]$, fuzzy planar graphs $[17,18]$, bipolar fuzzy hypergraphs $[19,20]$, and completeness and regularity of generalized fuzzy graphs [21].

One of the generalizations of FGs is intuitionistic fuzzy graph (IFG). The notion of IFG was presented by Shannon and Atanassov who also developed the idea of IFS relation, which was used in defining the IFGs [22]. They also discussed the many properties, theorems, and proofs regarding IFSs and IFGs. The work of Atanassov and Shannon was extended by many researchers who presented its properties, types, and applications; for example, join, union, and product of two IFGs were defined by Parvathi et al., and strong products, direct products, and lexicographic products of two IFGs were presented by Rashmanlou et al. The concepts of strong IFGs and intuitionistic fuzzy hypergraphs along with their applications were discussed by Akram and Davvaz [23, 24]. Karunambigai et al. discussed the balanced intuitionistic fuzzy graphs [25], covering and paired domination in IFGs [26], and intuitionistic fuzzy tolerance graph along with its applications was discussed by Sahoo and Pal $[27,28]$. Even though the FGS and IFGs are great tools to model real-world problems, some of the problems with uncertain, indeterminate, and varying information cannot be handled by FGs and IFGs. Therefore, some more efficient tool was required to tackle such problems, and this requirement led to the idea of NSs and NGs.

FSs and FGs discuss only the membership grades of the objects, while IFSs and IFGs discuss both the membership and nonmembership grades of an object, which do not totally model the human opinions, as human opinion can also be abstentious. Moreover, in IFSs and IFGs, the membership and nonmembership grades cannot be chosen independently. Their sum must be restricted to be in the unit interval. Therefore, this void created the space for the introduction of NSs and NGs. Smarandache, after initiating the concept of NSs, proposed four main categories of NGs: I-vertex NG and I-edge NG are based on literal indeterminacy $(I)$, while $(t, I, f)$-vertex NG and $(t, I, f)$-edge $N G$ are based on (t, I, f) components [29-33]. In NSs and NGs, the membership, abstinence, and nonmembership grades can be chosen independently from the unit interval, which provides comfort and ease to a decision-maker. Lately, NGs caught the attention of many researchers [34-40]. In addition, Garg [41-43] introduced aggregation operators and multiple decision-making techniques via SVNSs.

This study focuses on the concepts of SVNGs, which were initiated by Broumi and Smarandache by applying the concept of SVNS to graphs [44]. They also discussed different types of SVNGs, bipolar SVNGs [45], grade, order, and size [46] and investigated many properties with proofs and examples. Recently, some researchers have worked on SVNGs, for instance, operations on SVNGs presented by Akram and Shahzadi [47], applications of operations on SVNGs given by Naz et al. [36], and properties of SVNGs discussed by Karaaslan and Davvaz [48].

Since the domain of SVNG is greater than that of FGs and IFGs, it expands the range of applications of graphs. Like all other graphs, SVNGs also model the relations. So, it is applied to all sorts of problems consisting of relationships. It is capable of modeling the problems with uncertain and varying information in real world, where FGs and IFGs fail. This article introduces the notions of covering, matching, and paired domination in SVNG, complete SVNGs, and complete bipartite SVNGs. Moreover, strong coverings, that is, strong vertex cover and strong edge cover, the strong vertex covering number, and strong edge covering numbers are defined using the strong edge. In addition, the strong independent sets, the strong independent number, strong matching, and strong matching number are also discussed along with some interesting properties, theorems, proofs, and examples. The paired domination in SVNGs is also explained with examples and theorems.

The paper is organized as follows: Section 2 reviews some basic definitions and examples of FGs, IFSs, IFRs, IFGs, SVNSs, SVNRs, and SVNGs. Section 3 defines the strong covering of vertices and edges, strong independent sets (SIS), and strong matchings (SM) using strong edges (SEs) along with suitable examples and several interesting properties. Section 4 introduces the paired domination (PD), strong paired domination (SPD), and perfect paired domination. Finally, the research is concluded in Section 5.

\section{Preliminaries}

This section recalls some basic definitions of fuzzy graph (FG), intuitionistic fuzzy set (IFS), intuitionistic fuzzy relation (IFR), intuitionistic fuzzy graph (IFG), single-valued neutrosophic set (SVNS), single-valued neutrosophic relation (SVNR), and single-valued neutrosophic graph (SVNG). In addition, the examples of graphs are also given for better understanding.

Definition 1 (see [8]). A pair $G=(V, E)$ is said to be an FG, where $V$ is an FS in universe $\ddot{X}$ defined as $V=\left\{v_{1}, v_{2}, v_{3}, \ldots, v_{n}\right\}$ and called the collection of vertices possessing the grade of membership $m_{1}: V \longrightarrow[0,1]$ and the collection $E$ on $\ddot{\mathrm{X}}$ is defined as

$E \subseteq V \times V=\left\{\left(v_{i}, v_{j}\right) \mid v_{i}, v_{j} \in V, i, j=1,2,3, \ldots, n\right\} \quad$ and called the collection of edges possessing the grade of membership $m_{2}: V \times V \longrightarrow[0,1]$, such that 
$m_{2}\left(v_{i}, v_{j}\right) \leq \min \left\{m_{1}\left(v_{i}\right), m_{1}\left(v_{j}\right)\right\}, \quad \forall v_{i}, v_{j} \in V,\left(v_{i}, v_{j}\right) \in E$.

Example 1. An illustration of an $\mathrm{FG} G=(V, E)$ is given in Figure 1. Here, the collections of vertices and edges are $V=$ $\left\{v_{1}, v_{2}, v_{3}, v_{4}, v_{5}\right\}$ and $E=\left\{\left(v_{1}, v_{2}\right),\left(v_{1}, v_{3}\right),\left(v_{1}, v 4\right),\left(v_{1}\right.\right.$, $\left.\left.v_{5}\right),\left(v_{2}, v_{3}\right),\left(v_{2}, v_{4}\right),\left(v_{2}, v_{5}\right),\left(v_{3}, v_{4}\right),\left(v_{3}, v_{5}\right),\left(v_{4}, v_{5}\right)\right\}$, respectively.

Definition 2 (see [3]). A nonempty collection $A$ in universe $\ddot{X}$ of the following form is called an IFS:

$$
A=\{<m(\ddot{x}), n(\ddot{x})>, \ddot{x} \in A\},
$$

where the mapping $m: A \longrightarrow[0,1]$ represents the grade of membership and the mapping $n: A \longrightarrow[0,1]$ represents the nonmembership of $A$, such that

$$
m(\ddot{x})+n(\ddot{x}) \in[0,1] .
$$

Definition 3 (see [3]). Let $A_{1}=\left(m_{1}, n_{1}\right)$ and $A_{2}=\left(m_{2}, n_{2}\right)$ be two IFSs such that $m_{1}: A_{1} \longrightarrow[0,1]$ and $n_{1}: A_{1} \longrightarrow[0,1]$ are mappings representing the membership and nonmembership grades of $A_{1}$, respectively, and $m_{2}: A_{2} \longrightarrow[0,1]$ and $n_{2}: A_{2} \longrightarrow[0,1]$ are mappings representing the membership and nonmembership grades of $A_{2}$, respectively; then the intuitionistic fuzzy relation (IFR) denoted by $\bar{R}$ is a collection of the form

$\bar{R} \cdot=A_{1} \times A_{2}=\left\{<(\ddot{x}, \ddot{y}), m(\ddot{x}, \ddot{y}), n(\ddot{x}, \ddot{y})>\mid \ddot{x} \in A_{1}, \ddot{y} \in A_{2}\right\}$.

The mapping $m: A_{1} \times A_{2} \longrightarrow[0,1]$ represents the membership grade and the mapping $n: A_{1} \times A_{2} \longrightarrow[0,1]$ represents the nonmembership grade of $\bar{R}$, where

$$
\begin{aligned}
& m(x, \ddot{y}) \leq \min \left\{m_{1}(\ddot{x}), m_{1}(\ddot{y})\right\}, \quad \leq \max \left\{n_{1}(\ddot{x}), n_{1}(\ddot{y})\right\} . \\
& n(x, \ddot{y}
\end{aligned}
$$

This is provided that $m(x, \ddot{y})+n(x, \ddot{y}) \in[0,1]$.

Definition 4 (see [22]). An IFG is a pair $G=(V, E)$, where $V$ is an IFS in universe $\ddot{X}$ defined as

$V=\left\{v_{1}, v_{2}, v_{3}, \ldots, v_{n}\right\}$ and called the collection of vertices possessing the membership grade $m_{1}: V \longrightarrow[0,1]$ and nonmembership grade $n_{1}: V \longrightarrow[0,1]$, such that

$$
m_{1}\left(v_{i}\right)+n_{1}\left(v_{i}\right) \in[0,1], \quad \forall v_{i} \in V,
$$

and the collection $E$ on $\ddot{X}$ is defined as $E \subseteq V \times V=\left\{\left(v_{i}, v_{j}\right) \mid v_{i}, v_{j} \in V, i, j=1,2,3, \ldots, n\right\}$ and called the collection of edges possessing the membership grade $m_{2}: V \times V \longrightarrow[0,1]$ and the nonmembership grade $n_{2}: V \times V \longrightarrow[0,1]$, such that

$$
\begin{gathered}
m_{2}\left(v_{i}, v_{j}\right) \leq \min \left\{m_{1}\left(v_{i}\right), m_{1}\left(v_{j}\right)\right\} \\
n_{2}\left(v_{i}, v_{j}\right) \leq \max \left\{n_{1}\left(v_{i}\right), n_{1}\left(v_{j}\right)\right\} .
\end{gathered}
$$

This is provided that $m_{2}\left(v_{i}, v_{j}\right)+n_{2}\left(v_{i}, v_{j}\right) \in[0,1]$, $\forall\left(v_{i}, v_{j}\right) \in E$.

Example 2. In Figure 2, the collection of vertices and the collection of edges for an IFG $G$ are $V=\left\{v_{1}, v_{2}, v_{3}, v_{4}\right\}$ and $E=\left\{\left(v_{1}, v_{2}\right),\left(v_{1}, v_{3}\right),\left(v_{2}, v_{4}\right),\left(v_{3}, v_{4}\right)\right\}$, respectively.

Definition 5 (see [7]). A nonempty collection $A$ in universe $\ddot{X}$ of the following form is called an SVNS:

$$
A=\{<m(\ddot{x}), \alpha(\ddot{x}), n(\ddot{x})>, \ddot{x} \in A\},
$$

where the mapping $m: A \longrightarrow[0,1]$ represents the membership grade, $\alpha: A \longrightarrow[0,1]$ represents the abstinence grade, and $n: A \longrightarrow[0,1]$ represents the nonmembership grade such that

$$
m(\ddot{x})+\alpha(\ddot{x})+n(\ddot{x}) \in[0,3] .
$$

Definition 6 (see [46]). Let $A_{1}=\left(m_{1}, \alpha_{1}, n_{1}\right)$ and $A_{2}=\left(m_{2}, \alpha_{2}, n_{2}\right)$ be two SVNSs; then, $A_{1}$ is said to be the SVNR on $A_{2}$, if $\forall \ddot{x}, \ddot{y} \in A_{1}$,

$$
\begin{aligned}
& m(x, \ddot{y}) \leq \min \left\{m_{1}(\ddot{x}), m_{1}(\ddot{y})\right\}, \\
& \alpha(x, \ddot{y}) \leq \min \left\{\alpha_{1}(\ddot{x}), \alpha_{1}(\ddot{y})\right\}, \\
& n(x, \ddot{y}) \leq \max \left\{n_{1}(\ddot{x}), n_{1}(\ddot{y})\right\} .
\end{aligned}
$$

This is provided that $m(\ddot{x}, \ddot{y})+\alpha(\ddot{x}, \ddot{y})+n(\ddot{x}, \ddot{y}) \epsilon$ $[0,3]$, where $m_{1}, \alpha_{1}$, and $n_{1}$ are the membership, abstinence, and nonmembership grades of $A_{1}$, respectively, and $m_{2}, \alpha_{2}$, and $n_{2}$ are the membership, abstinence, and nonmembership grades of $A_{2}$, respectively.

Definition 7 (see [44]). An SVNG is a pair $G=(V, E)$, where $V$ is an SVNS in $\ddot{X}$ defined as $V=\left\{v_{1}, v_{2}, v_{3}, \ldots, v_{n}\right\}$ and called the collection of vertices possessing the membership grade $m_{1}: V \longrightarrow[0,1]$, abstinence grade $\alpha_{1}: V \longrightarrow[0,1]$, and nonmembership grade $n_{1}: V \longrightarrow[0,1]$, such that

$$
m_{1}\left(v_{i}\right)+\alpha_{1}\left(v_{i}\right)+n_{1}\left(v_{i}\right) \in[0,3], \quad \forall v_{i} \in V,
$$

and $E \subseteq V \times V=\left\{\left(v_{i}, v_{j}\right) \mid v_{i}, v_{j} \in V, i, j=1,2,3, \ldots, n\right\} \quad$ is called the collection of edges possessing the membership grade $m_{2}: V \times V \longrightarrow[0,1], \quad$ abstinence grade $\alpha_{2}: V \times V \longrightarrow[0,1]$, and nonmembership grade $n_{2}: V \times V \longrightarrow[0,1]$, such that

$$
\begin{aligned}
& m_{2}\left(v_{i}, v_{j}\right) \leq \min \left\{m_{1}\left(v_{i}\right), m_{1}\left(v_{j}\right)\right\}, \\
& \alpha_{2}\left(v_{i}, v_{j}\right) \leq \min \left\{\alpha_{1}\left(v_{i}\right), \alpha_{1}\left(v_{j}\right)\right\}, \\
& n_{2}\left(v_{i}, v_{j}\right) \leq \max \left\{n_{1}\left(v_{i}\right), n_{1}\left(v_{j}\right)\right\} .
\end{aligned}
$$

This is provided that $m_{2}\left(v_{i}, v_{j}\right)+\alpha_{2}\left(v_{i}, v_{j}\right)+n_{2}\left(v_{i}, v_{j}\right) \in$ $[0,1], \forall\left(v_{i}, v_{j}\right) \in E$.

Example 3. An SVNG $G=(V, E)$ is constructed in Figure 3 using the collection of vertices $V=\left\{v_{1}, v_{2}, v_{3}, v_{4}\right\}$ and the collection of edges $E=\left\{\left(v_{1}, v_{2}\right),\left(v_{1}, v_{3}\right),\left(v_{2}, v_{4}\right),\left(v_{3}, v_{4}\right)\right\}$. 


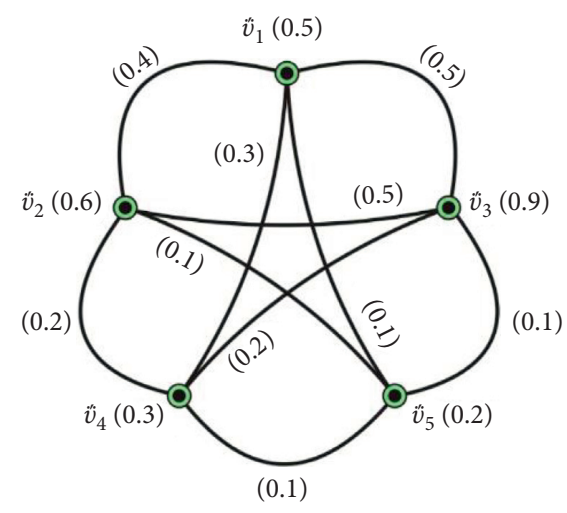

Figure 1: Fuzzy graph.

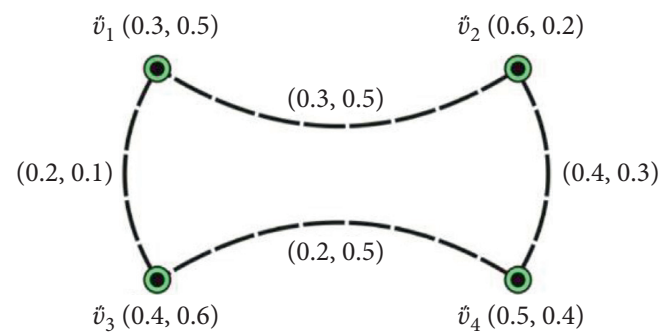

FIgURe 2: Intuitionistic fuzzy graph.

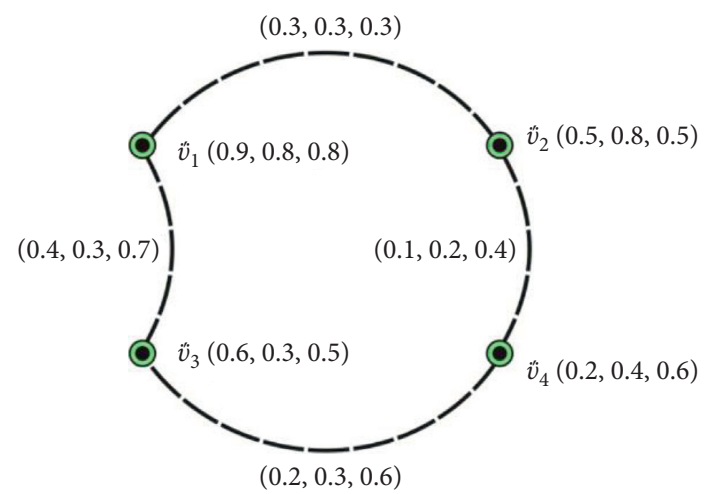

FIGURE 3: Single-valued neutrosophic graph.

\section{Covering and Matching in Single-Valued Neutrosophic Graphs}

This section presents the definitions and examples of strong covering of vertices and edges, strong independent sets (SIS), and strong matchings (SM) using strong edges (SEs). Some interesting theorems are also proved for covering and matching in complete SVNG, SVNC, and complete bipartite SVNG.

Definition 8. Let $G=(V, E)$ be an SVNG. A vertex and an $\mathrm{SE}$ incident to it are said to strongly cover each other. The collection of vertices $\mathbf{S}$ which covers all the SEs of an SVNG $G$ is known as strong vertex cover (SVC) in $G . M_{1}(\dot{S})=$ $\sum_{u \in \dot{S}} m_{2}(u, v)$ is the membership grade, $M_{2}(\dot{S})=\sum_{u \in \dot{S}}$ $\alpha_{2}(u, v)$ is the abstinence grade, and $M_{3}(\dot{S})=\sum_{u \in \dot{S}} n_{2}(u, v)$ is the nonmembership grade values of the SVC $S$, where $m_{2}(u, v)$ and $\alpha_{2}(u, v)$ are the minimum of the membership and the abstinence values, respectively, and $n_{2}(u, v)$ is the maximum value among the collection of the nonmembership values of the strong edges incident on $u$. Let $C_{10}$ and $C_{20}$ denote the minimum values of membership and abstinence, and let $C_{30}$ denote the maximum value of nonmembership of the SVC of $G$. Then the strong vertex covering number of $G$ is defined and denoted by $C_{0}(G)=C_{0}=\left(C_{10}, C_{20}, C_{30}\right)$. An SVC with minimum membership value, minimum abstinence value, and maximum nonmembership value in an SVNG $G$ is said to be a minimum SVC.

Theorem 1. Let $G=(V, E)$ be a complete SVNG; then, $C_{10}, C_{20}$, and $C_{30}$ are defined as

$$
\begin{aligned}
& C_{10}=(n-1) m_{2}(u, v), \\
& C_{20}=(n-1) \alpha_{2}(u, v), \\
& C_{30}=(n-1) n_{2}(u, v),
\end{aligned}
$$

where $m_{2}(u, v)$ is the membership value, $\alpha_{2}(u, v)$ the abstinence value, and $n_{2}(u, v)$ is the nonmembership value of the weakest edge in $G$, and $n$ is the number of vertices in $G$.

Proof. Since $G=(V, E)$ is a complete SVNG, each of its vertices is connected to every other vertex in $G$ and all of its edges are strong. Hence, a strong cover vertex of $G$ is formed by any collection of $(n-1)$ vertices. Let $u$ be a minimum SVC, and $u$ is connected to the vertices $v_{1}, v_{2}, v_{3}, \ldots, v_{n-1}$. Then, the edges $\left(u, v_{1}\right),\left(u, v_{2}\right),\left(u, v_{3}\right), \ldots,\left(u, v_{n-1}\right)$ are all the weakest edges of $G$ with $m_{2}(u, v)$ as the membership strength, $\alpha_{2}(u, v)$ as the abstinence strength, and $n_{2}(u, v)$ as the nonmembership strength, where the collection $\dot{S} \in\left\{v_{1}, v_{2}, v_{3}, \ldots, v_{n-1}\right\}$ of $n-1$ vertices forms an SVC of $G$ with

$$
M_{1}(\dot{S})=\sum_{v_{i} \in \dot{S}} m_{2}\left(u, v_{i}\right), \quad i=1,2,3, \ldots,(n-1),
$$

where $m_{2}\left(u, v_{i}\right)$ is the minimum of the membership value of the SEs incident on $v_{i}$. If the value of membership for the weakest edge of graph $G$ is represented by $m_{2}(u, v)$, then

$$
\begin{aligned}
C_{10}= & m_{2}(u, v)+m_{2}(u, v)+m_{2}(u, v) \\
& +\cdots+m_{2}(u, v)[(n-1) \text { times }] .
\end{aligned}
$$

Therefore, $C_{10}=(n-1) m_{2}(u, v)$.

Now,

$$
M_{2}(\dot{S})=\sum_{v_{i} \in \dot{S}} \alpha_{2}\left(u, v_{i}\right), \quad i=1,2,3, \ldots,(n-1),
$$

where $m_{2}\left(u, v_{i}\right)$ is the minimum of the abstinence value of the $\mathrm{SE}$ incident on $v_{i}$. If the value of abstinence for the weakest edge of graph $G$ is represented by $\alpha_{2}(u, v)$, then

$$
\begin{aligned}
C_{20}= & \alpha_{2}(u, v)+\alpha_{2}(u, v)+\alpha_{2}(u, v) \\
& +\cdots+\alpha_{2}(u, v)[(n-1) \text { times }] .
\end{aligned}
$$

Therefore, $C_{20}=(n-1) \alpha_{2}(u, v)$.

In the same way, 


$$
M_{3}(\dot{S})=\sum_{v_{i} \in \dot{S}} n_{2}\left(u, v_{i}\right), \quad i=1,2,3, \ldots,(n-1),
$$

where $n_{2}\left(u, v_{i}\right)$ is the maximum of the nonmembership value of the SE incident on $v_{i}$. If the value of abstinence for the weakest edge of graph $G$ is represented by $n_{2}(u, v)$, then

$$
C_{30}=n_{2}(u, v)+n_{2}(u, v)+n_{2}(u, v)+\cdots+n_{2}(u, v)[(n-1) \text { times }] .
$$

Therefore, $C_{30}=(n-1) n_{2}(u, v)$.

Theorem 2. If $K$ is a complete bipartite $S V N G$ with $V_{1}$ and $V_{2}$ as partite sets, then

$$
\begin{aligned}
& C_{10}(K)=\min \left\{M_{1}\left(V_{1}\right), M_{1}\left(V_{2}\right)\right\}, \\
& C_{20}(K)=\min \left\{M_{2}\left(V_{1}\right), M_{1}\left(V_{2}\right)\right\}, \\
& C_{30}(K)=\max \left\{M_{3}\left(V_{1}\right), M_{3}\left(V_{2}\right)\right\} .
\end{aligned}
$$

Proof. Since $K$ is a complete bipartite SVNG, all of its edges are strong. Moreover, each of the vertices in $V_{1}$ is connected to all the vertices in $V_{2}$ and each of the vertices in $V_{2}$ is connected to all the vertices of $V_{1}$. The collection of all edges of $K$ is the union of the collection of all edges incident to every vertex in $V_{1}$ and the collection of all edges incident to each vertex in $V_{2}$. Therefore, $V_{1}, V_{2}$, and $V_{1} \cup V_{1}$ are the SVCs in $K$. It is obvious that

$$
\begin{aligned}
& M_{1}\left(V_{1} \cup V_{2}\right)>M_{1}\left(V_{1}\right), \\
& M_{1}\left(V_{1} \cup V_{2}\right)>M_{1}\left(V_{2}\right) .
\end{aligned}
$$

Therefore, $C_{10}(K)=\min \left\{M_{1}\left(V_{1}\right), M_{1}\left(V_{2}\right)\right\}$.

And, $\quad M_{2}\left(V_{1} \cup V_{2}\right)>M_{2}\left(V_{1}\right)$ and $M_{2}\left(V_{1} \cup V_{2}\right)>$ $M_{2}\left(V_{2}\right)$.

Therefore, $C_{20}(K)=\min \left\{M_{2}\left(V_{1}\right), M_{1}\left(V_{2}\right)\right\}$.

Similarly, $M_{3}\left(V_{1} \cup V_{2}\right)<M_{3}\left(V_{1}\right)$ and $M_{3}\left(V_{1} \cup V_{2}\right)>$ $M_{3}\left(V_{2}\right)$.

Therefore, $C_{30}(K)=\max \left\{M_{3}\left(V_{1}\right), M_{3}\left(V_{2}\right)\right\}$.

Theorem 3. If $G=(V, E)$ is a single-valued neutrosophic cycle (SVNC) and $\bar{g}$ is a crisp cycle (CC), then

$$
\begin{aligned}
& C_{10}(G)=\min \left\{M_{1}(\dot{S}) \mid \dot{S} \text { is an SVC in } G \text { with }|\dot{S}| \geq \frac{n}{2}\right\}, \\
& C_{20}(G)=\min \left\{M_{2}(\dot{S}) \mid \dot{S} \text { is an SVC in } G \text { with }|\dot{S}| \geq \frac{n}{2}\right\}, \\
& C_{30}(G)=\max \left\{M_{3}(\dot{S}) \mid \dot{S} \text { is an SVC in } G \text { with }|\dot{S}| \geq \frac{n}{2}\right\} .
\end{aligned}
$$

Proof. As every edge in an SVNC is strong, the SVC number of $G$ is $(n / 2)$, since the number of strong vertices in SVNG and the crisp cycle $\bar{g}$ are the same because each edge is strong in both graphs [49]. Therefore, in the SVC of $G,(n / 2)$ is the least number of vertices. So,

$$
\begin{aligned}
& C_{10}(G)=\min \left\{M_{1}(\dot{S}) \mid \dot{S} \text { is an SVC in } G \text { with }|\dot{S}| \geq \frac{n}{2}\right\}, \\
& C_{20}(G)=\min \left\{M_{2}(\dot{S}) \mid \dot{S} \text { is an SVC in } G \text { with }|\dot{S}| \geq \frac{n}{2}\right\}, \\
& C_{30}(G)=\max \left\{M_{3}(\dot{S}) \mid \dot{S} \text { is an SVC in } G \text { with }|\dot{S}| \geq \frac{n}{2}\right\} .
\end{aligned}
$$

Definition 9. Two vertices are said to be strongly independent in an SVNG $G=(V, E)$ if they are not connected by an SE. If any collection in $G$ contains any two strongly independent vertices, then such a collection is known as strong independent set (SIS).

Definition 10. Let $S$ be an SIS in an SVNG $G=(V, E)$. Then the values of membership, abstinence, and nonmembership of $\dot{S}$ are defined as $M_{1}(\dot{S})=\sum_{u \in \dot{S}} m_{2}(u, v), M_{2}(\dot{S})=\sum_{u \in \dot{S}} \alpha_{2}$ $(u, v)$, and $M_{3}(\dot{S})=\sum_{u \in \dot{S}} n_{2}(u, v)$, respectively, where $m_{2}(u, v)$ and $\alpha_{2}(u, v)$ represent the least value among the values of membership and the least value among the values of abstinence of the SEs incident on $u$. Similarly, the nonmembership value of $S$ is defined, where $n_{2}(u, v)$ represents the greatest value among the values of nonmembership of the SEs incident on $u$.

$D_{0}(G)=D_{0}=\left(D_{10}, D_{20}, D_{30}\right)$ denotes and defines the strong independent number of an SVNG $G=(V, E)$, where $D_{10}$ and $D_{20}$ are greatest values of membership and greatest values of abstinence of $S$ in $G$, respectively, and $D_{30}$ is the least value of nonmembership of $S$ in $G$. The SIS with maximum membership value, maximum abstinence value, and minimum nonmembership value in an SVNG $G$ is known as a maximum SIS of vertices.

Theorem 4. If $G=(V, E)$ is a complete $S V N G$, then $D_{10}=m_{2}(u, v), D_{20}=\alpha_{2}(u, v)$, and $D_{30}=n_{2}(u, v)$, where $m_{2}(u, v), \alpha_{2}(u, v)$, and $n_{2}(u, v)$ are the membership, abstinence, and nonmembership values of the weakest edge in $G$.

Proof. Since $G=(V, E)$ is a complete SVNG, each of its vertices is connected to every other vertex in $G$ and all of its edges are strong. Therefore, there is only single SIS, that is, $\dot{S}=\{u\}$. Hence, the result follows.

Theorem 5. If $K$ is a complete bipartite $S V N G$ with $V_{1}$ and $V_{2}$ as partite sets, then

$$
\begin{aligned}
& D_{10}(K)=\max \left\{M_{1}\left(V_{1}\right), M_{1}\left(V_{2}\right)\right\}, \\
& D_{20}(K)=\max \left\{M_{2}\left(V_{1}\right), M_{1}\left(V_{2}\right)\right\}, \\
& D_{30}(K)=\min \left\{M_{3}\left(V_{1}\right), M_{3}\left(V_{2}\right)\right\} .
\end{aligned}
$$

Proof. Since $K$ is a complete bipartite SVNG, all of its edges are strong. Moreover, each of the vertices in $V_{1}$ is connected to all the vertices in $V_{2}$, and each of the vertices in $V_{2}$ is connected to all the vertices of $V_{1}$. Therefore, $V_{1}$ and $V_{2}$ are the SISs in $K$. Hence, 


$$
\begin{aligned}
& D_{10}(K)=\max \left\{M_{1}\left(V_{1}\right), M_{1}\left(V_{2}\right)\right\}, \\
& D_{20}(K)=\max \left\{M_{2}\left(V_{1}\right), M_{1}\left(V_{2}\right)\right\}, \\
& D_{30}(K)=\min \left\{M_{3}\left(V_{1}\right), M_{3}\left(V_{2}\right)\right\} .
\end{aligned}
$$

Theorem 6. Let $G=(V, E)$ be an SVNC and $\bar{g}$ denotes a $C C$, then

$$
\begin{aligned}
& D_{10}(G)=\max \left\{M_{1}(\dot{S}) \mid \dot{S} \text {.is an SVC in } G \text { with }|\dot{S}| \leq \frac{n}{2}\right\}, \\
& D_{20}(G)=\max \left\{M_{2}(\dot{S}) \mid \dot{S} \text {.is an SVC in } G \text { with }|\dot{S}| \leq \frac{n}{2}\right\}, \\
& D_{30}(G)=\min \left\{M_{3}(\dot{S}) \mid \dot{S} \text {.is an SVC in } G \text { with }|\dot{S}| \leq \frac{n}{2}\right\} .
\end{aligned}
$$

Proof. As every edge in an SVNC is strong, the SVC number of $G$ is $n / 2$, since the number of vertices in a SISs in $\bar{g}$ and $G$ are the same because each edge is strong in both graphs [49]. So, in the SVC of $G, n / 2$ is the greatest number of vertices. Thus,

$$
\begin{aligned}
& D_{10}(G)=\max \left\{M_{1}(\dot{S}) \mid \dot{S} \text { is an SVC in } G \text { with }|\dot{S}| \leq \frac{n}{2}\right\}, \\
& D_{20}(G)=\max \left\{M_{2}(\dot{S}) \mid \dot{S} \text { is an SVC in } G \text { with }|\dot{S}| \leq \frac{n}{2}\right\}, \\
& D_{30}(G)=\min \left\{M_{3}(\dot{S}) \mid \dot{S} \text { is an SVC in } G \text { with }|\dot{S}| \leq \frac{n}{2}\right\} .
\end{aligned}
$$

Proof. As each of the vertices in a complete SVNG $G$ is connected to every other vertex of $G$ and all of its edges are strong, the SEC number of $G$ is $n / 2$, since each edge is strong in a complete SVNG and crisp graph; therefore, the numbers of strong edges in both graphs are the same [49]. Hence, the least number of edges in an SEC in $G$ is $n / 2$. Hence,

$$
\begin{aligned}
& C_{11}(G)=\min \left\{M_{1}(\dot{T}) \mid \dot{T} \text { is an SEC in } G \text { with }|\dot{T}| \geq \frac{n}{2}\right\}, \\
& C_{21}(G)=\min \left\{M_{2}(\dot{T}) \mid \dot{T} \text { is an SEC in } G \text { with }|\dot{T}| \geq \frac{n}{2}\right\}, \\
& C_{31}(G)=\max \left\{M_{3}(\dot{T}) \mid \dot{T} \text { is an SEC in } G \text { with }|\dot{T}| \geq \frac{n}{2}\right\}
\end{aligned}
$$

Theorem 8. If $K$ is a complete bipartite SVNG with $V_{1}$ and $V_{2}$ as partite sets, then

$$
\begin{aligned}
& C_{11}(K)=\min \left\{M_{1}(\dot{T}) \mid \dot{T} \text { is an SEC in } K \text { with }|\dot{T}| \geq \max \left\{\left|V_{1}\right|,\left|V_{2}\right|\right\}\right\}, \\
& C_{21}(K)=\min \left\{M_{2}(\dot{T}) \mid \dot{T} \text { is an SEC in } K \text { with }|\dot{T}| \geq \max \left\{\left|V_{1}\right|,\left|V_{2}\right|\right\}\right\}, \\
& C_{31}(K)=\max \left\{M_{3}(\dot{T}) \mid \dot{T} \text { is an SEC in } K \text { with }|\dot{T}| \geq \max \left\{\left|V_{1}\right|,\left|V_{2}\right|\right\}\right\} .
\end{aligned}
$$


Proof. Since $K$ is complete bipartite SVNG, all of its edges are strong. Moreover, each of the vertices in $V_{1}$ is connected to all the vertices in $V_{2}$ and vice versa. The edge covering number of $K$ is $\left\{\left|V_{1}\right|,\left|V_{2}\right|\right\}$, since each edge is strong in a complete bipartite SVNG and $\bar{g}$; therefore, the number of SEs in both graphs is the same [49]. Hence, the least number of edges in the SEC in $K$ is $\left\{\left|V_{1}\right|,\left|V_{2}\right|\right\}$. Thus,

$$
\begin{aligned}
& C_{11}(K)=\min \left\{M_{1}(\dot{T}) \mid \dot{T} \text { is an SEC in } K \text { with }|\dot{T}| \geq \max \left\{\left|V_{1}\right|,\left|V_{2}\right|\right\}\right\}, \\
& C_{21}(K)=\min \left\{M_{2}(\dot{T}) \mid \dot{T} \text { is an SEC in } K \text { with }|\dot{T}| \geq \max \left\{\left|V_{1}\right|,\left|V_{2}\right|\right\}\right\}, \\
& C_{31}(K)=\max \left\{M_{3}(\dot{T}) \mid \dot{T} \text { is an SEC in } K \text { with }|\dot{T}| \geq \max \left\{\left|V_{1}\right|,\left|V_{2}\right|\right\}\right\} .
\end{aligned}
$$

Theorem 9. Let $G=(V, E)$ be an SVNC and $\bar{g}$ is a $C C$, then

$$
\begin{aligned}
& C_{11}(G)=\min \left\{M_{1}(\dot{T}) \mid \dot{T} \text { is an SEC in } K \text { with }|\dot{T}| \geq \frac{n}{2}\right\}, \\
& C_{21}(G)=\min \left\{M_{2}(\dot{T}) \mid \dot{T} \text { is an SEC in } K \text { with }|\dot{T}| \geq \frac{n}{2}\right\}, \\
& C_{31}(G)=\max \left\{M_{3}(\dot{T}) \mid \dot{T} \text { is an SEC in } K \text { with }|\dot{T}| \geq \frac{n}{2}\right\} .
\end{aligned}
$$

Proof. Since every edge in an SVNC is strong, the SEC number of $G$ is $n / 2$, as the number of SEs in SVNG and the crisp cycle $\bar{g}$ are the same because each edge is strong in both graphs [49]. So, in the SEC of $G, n / 2$ is the least number of edges. Thus,

$$
\begin{aligned}
& C_{11}(G)=\min \left\{M_{1}(\dot{T}) \mid \dot{T} \text { is an SEC in } K \text { with }|\dot{T}| \geq \frac{n}{2}\right\}, \\
& C_{21}(G)=\min \left\{M_{2}(\dot{T}) \mid \dot{T} \text { is an SEC in } K \text { with }|\dot{T}| \geq \frac{n}{2}\right\}, \\
& C_{31}(G)=\max \left\{M_{3}(\dot{T}) \mid \dot{T} \text { is an SEC in } K \text { with }|\dot{T}| \geq \frac{n}{2}\right\} .
\end{aligned}
$$

Definition 12. A collection of SEs denoted by Ö̈ in an SVNG $G=(V, E)$ is said to be an SIS of edges if all of its edges do not share a vertex. Ö is also known as strong matching (SM) in $G=(V, E)$.

Definition 13. If $(u, v) \in \ddot{\widetilde{O}}$, where $\widetilde{O}$ is an SM in an SVNG $G=(V, E)$, then it is said that $u$ is strongly matched to $v$ by O. $\quad M_{1}(\ddot{\widetilde{O}})=\sum_{(u, v) \in \tilde{\widetilde{O}}} m_{2}(u, v), \quad M_{2}(\ddot{\widetilde{O}})=\sum_{(u, v) \in \in} \ddot{\widetilde{O}} \alpha_{2}(u, v)$, and $M_{3}(\ddot{\widetilde{O}})=\sum_{(u, v) \in \tilde{\tilde{O}}} n_{2}(u, v)$ are the membership, abstinence, and nonmembership values of the SEC O, respectively.

$C_{1}(G)=C_{1}=\left(C_{11}, C_{21}, C_{31}\right)$ is the strong edge independent number or SM number of an SVNG $G$, where $C_{11}$ and $C_{21}$ are the maximum membership and abstinence values of the SMs of $G$, and $C_{31}$ denotes the minimum nonmembership value. An SM with maximum membership value, maximum abstinence value, and minimum nonmembership value in an SVNG $G$ is said to be a maximum SM.

Theorem 10. Let $G=(V, E)$ be an $S V N G$; then

$$
\begin{aligned}
& D_{11}(G)=\max \left\{M_{1}(\ddot{\widetilde{O}}) \mid \ddot{\widetilde{O}} \text { is an } S M \text { in } G \text { with }|\ddot{\widetilde{O}}| \leq \frac{n}{2}\right\}, \\
& D_{21}(G)=\max \left\{M_{2}(\ddot{\widetilde{O}}) \mid \ddot{\widetilde{O}} \text { is an } S M \text { in } G \text { with }|\ddot{\widetilde{O}}| \leq \frac{n}{2}\right\}, \\
& D_{31}(G)=\min \left\{M_{3}(\ddot{\widetilde{O}}) \mid \ddot{\widetilde{O}} \text { is an } S M \text { in } G \text { with }|\ddot{\widetilde{O}}| \leq \frac{n}{2}\right\},
\end{aligned}
$$

where $n$ denotes the number of vertices in $G=(V, E)$.

Proof. As each vertex of a complete SVNG $G$ is connected to every other vertex in $G$ and all of its edges are strong, the SM number of $G$ is $n / 2$, since each edge is strong in a complete SVNG and crisp graph; therefore the SM numbers in both graphs are the same [49]. So, in the SM of $G, n / 2$ is the greatest number of edges. Thus,

$$
\begin{aligned}
& D_{11}(G)=\max \left\{M_{1}(\ddot{\widetilde{O}}) \mid \ddot{\widetilde{O}} \text { is an SM in } G \text { with }|\ddot{\widetilde{O}}| \leq \frac{n}{2}\right\}, \\
& D_{21}(G)=\max \left\{M_{2}(\ddot{\widetilde{O}}) \mid \ddot{\widetilde{O}} \text { is an } S M \text { in } G \text { with }|\ddot{\widetilde{O}}| \leq \frac{n}{2}\right\}, \\
& D_{31}(G)=\min \left\{M_{3}(\ddot{\widetilde{O}}) \mid \ddot{\widetilde{O}} \text { is an SM in } G \text { with }|\ddot{\widetilde{O}}| \leq \frac{n}{2}\right\} .
\end{aligned}
$$

Theorem 11. If $K$ is a complete bipartite $S V N G$ with $V_{1}$ and $V_{2}$ as partite sets, then 


$$
\begin{aligned}
& D_{11}(G)=\max \left\{M_{1}(\ddot{\widetilde{O}}) \mid \ddot{\widetilde{O}} \text { is an SM in } K \text { with }|\ddot{\widetilde{O}}| \leq \min \left\{\left|V_{1}\right|,\left|V_{2}\right|\right\}\right\}, \\
& D_{21}(G)=\max \left\{M_{2}(\ddot{\widetilde{O}}) \mid \ddot{\widetilde{O}} \text { is an SM in } K \text { with }|\ddot{\widetilde{O}}| \leq \min \left\{\left|V_{1}\right|,\left|V_{2}\right|\right\}\right\}, \\
& D_{31}(G)=\min \left\{M_{3}(\ddot{\widetilde{O}}) \mid \ddot{\widetilde{O}} \text { is an SM in } K \text { with }|\ddot{\widetilde{O}}| \leq \min \left\{\left|V_{1}\right|,\left|V_{2}\right|\right\}\right\}
\end{aligned}
$$

Proof. Since $K$ is a complete bipartite SVNG, all of its edges are strong. Moreover, each of the vertices in $V_{1}$ is connected to all the vertices in $V_{2}$ and each of the vertices in $V_{2}$ is connected to all the vertices in $V_{1}$. The matching number of $K$ is $\left\{\left|V_{1}\right|,\left|V_{2}\right|\right\}$, since each edge is strong in a complete bipartite SVNG and complete bipartite crisp graph; therefore, the SM numbers in both graphs are the same [49]. So, in the SM of $K,\left\{\left|V_{1}\right|,\left|V_{2}\right|\right\}$ is the greatest number of edges. Thus,

$$
\begin{aligned}
& D_{11}(G)=\max \left\{M_{1}(\ddot{\widetilde{O}}) \mid \ddot{\widetilde{O}} \text { is an SM in } K \text { with }|\ddot{\widetilde{O}}| \leq \min \left\{\left|V_{1}\right|,\left|V_{2}\right|\right\}\right\}, \\
& D_{21}(G)=\max \left\{M_{2}(\ddot{\widetilde{O}}) \mid \ddot{\widetilde{O}} \text { is an SM in } K \text { with }|\ddot{\widetilde{O}}| \leq \min \left\{\left|V_{1}\right|,\left|V_{2}\right|\right\}\right\}, \\
& D_{31}(G)=\min \left\{M_{3}(\ddot{\widetilde{O}}) \mid \ddot{\widetilde{O}} \text { is an SM in } K \text { with }|\ddot{\widetilde{O}}| \leq \min \left\{\left|V_{1}\right|,\left|V_{2}\right|\right\}\right\} .
\end{aligned}
$$

Theorem 12. If $G=(V, E)$ is $S V N C$ and $\bar{g}$ is a $C C$, then

$$
\begin{aligned}
D(G) & =\max \left\{M_{1}(\ddot{\widetilde{O}}) \mid \ddot{\widetilde{O}} \text { is an } S M \text { in } G \text { with }|\ddot{\widetilde{O}}| \leq \frac{n}{2}\right\}, \\
D_{21}(G) & =\max \left\{M_{2}(\ddot{\widetilde{O}}) \mid \ddot{\widetilde{O}} \text { is an } S M \text { in } G \text { with }|\ddot{\widetilde{O}}| \leq \frac{n}{2}\right\}, \\
D_{31}(G) & =\min \left\{M_{3}(\ddot{\widetilde{O}}) \mid \ddot{\widetilde{O}} \text { is an } S M \text { in } G \text { with }|\ddot{\widetilde{O}}| \leq \frac{n}{2}\right\} .
\end{aligned}
$$

Proof. Every edge of an SVNC $G$ is strong. Moreover, $n / 2$ is the $S M$ number of $G$, and since each edge is strong in both of $G$ and $\bar{g}$, both graphs possess the same number of edges in SM [49]. So, in the SM of $G, n / 2$ is the greatest number of edges. Thus,

$$
\begin{aligned}
& D_{11}(G)=\max \left\{M_{1}(\ddot{\widetilde{O}}) \mid \ddot{\widetilde{O}} \text { is an } S M \text { in } G \text { with }|\ddot{\widetilde{O}}| \leq \frac{n}{2}\right\}, \\
& D_{21}(G)=\max \left\{M_{2}(\ddot{\widetilde{O}}) \mid \ddot{\widetilde{O}} \text { is an } S M \text { in } G \text { with }|\ddot{\widetilde{O}}| \leq \frac{n}{2}\right\}, \\
& D_{31}(G)=\min \left\{M_{3}(\ddot{\widetilde{O}}) \mid \ddot{\widetilde{O}} \text { is an } S M \text { in } G \text { with }|\ddot{\widetilde{O}}| \leq \frac{n}{2}\right\} .
\end{aligned}
$$

Example 4. Figure 4 displays an SVNG $G=(V, E)$. In $G$, the edges represented by solid lines $(a, \beta),(a, c)$, and $(c, d)$ are the SEs, while $(\beta, d)$ is not an SE, which is represented by dotted line. So, the SVCs are $\dot{S}_{1}=\{a, c\}, \dot{S}_{2}=\{a, d\}$, $\dot{S}_{3}=\{\beta, c\}, \quad \dot{S}_{4}=\{a, \beta, c\}, \quad \dot{S}_{5}=\{a, c, d\}, \quad \dot{S}_{6}=\{a, \beta, d\}$, $\dot{S}_{7}=\{\beta, c, d\}$, and $\dot{S}_{8}=\{a, \beta, c, d\}$.
Now, by the definition of the value of SVC,

$$
\begin{aligned}
M(\dot{\dot{S}}) & =\left(M_{1}(\dot{S}), M_{2}(\dot{S}), M_{3}(\dot{S})\right) \\
& =\left(\sum_{u \in \dot{S}} m_{2}(u, v), \sum_{u \in \dot{S}} \alpha_{2}(u, v), \sum_{u \in \dot{S}} n_{2}(u, v)\right),
\end{aligned}
$$

the following results are obtained for each SVC: $M\left(\dot{S}_{1}\right)=(0.2+0.2,0.4+0.4,0.7+0.8)=(0.4,0.8,1.5)$; similarly,

$$
\begin{aligned}
& M\left(\dot{S}_{2}\right)=(0.5,0.9,1.5), \\
& M\left(\dot{S}_{3}\right)=(0.8,1.1,1.0), \\
& M\left(\dot{S}_{4}\right)=(1.0,1.5,1.7), \\
& M\left(\dot{S}_{5}\right)=(0.7,1.3,2.3), \\
& M\left(\dot{S}_{6}\right)=(1.1,1.6,1.7), \\
& M\left(\dot{S}_{7}\right)=(1.1,1.6,1.8), \\
& M\left(\dot{S}_{8}\right)=(1.3,2.0,2.5) .
\end{aligned}
$$

The SVC number of $G$ is $C_{0}=\left(C_{10}, C_{20}, C_{30}\right)=(0.4,0.8,2.5)$. In this case, there is no minimum strong independent cover. In this case, there is no minimum SVC.

The vertices $a$ and $d$ are strongly independent since they are not linked by an SE. But they are not independent because are they adjacent.

The SISs are $\dot{S}_{1}=\{a, d\}, \dot{S}_{2}=\{\beta, d\}$, and $\dot{S}_{3}=\{\beta, c\}$. 


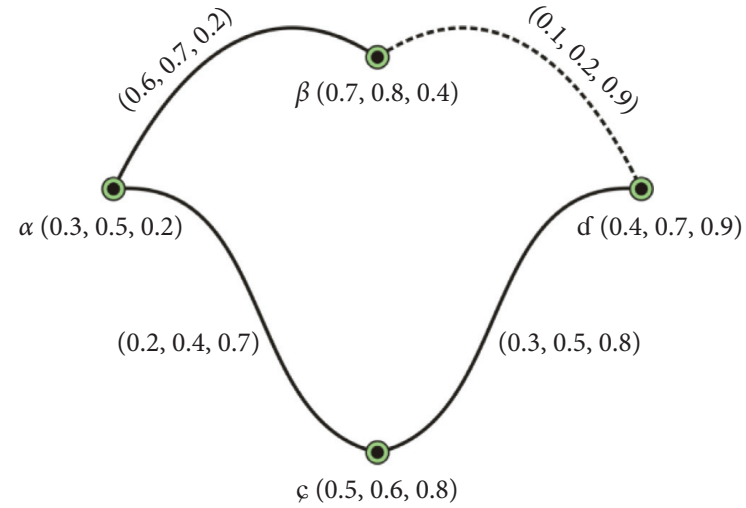

FIGURE 4: Strong covering in SVNG.

Now, the value of strong independent cover is calculated by using

$$
\begin{aligned}
M(\dot{S}) & =\left(M_{1}(\dot{S}), M_{2}(\dot{S}), M_{3}(\dot{S})\right) \\
& =\left(\sum_{u \in \dot{S}} m_{2}(u, v), \sum_{u \in \dot{S}} \alpha_{2}(u, v), \sum_{u \in \dot{S}} n_{2}(u, v)\right) .
\end{aligned}
$$

Thus,

$M\left(\dot{S}_{1}\right)=(0.2+0.3,0.4+0.5,0.7+0.8)=(0.5,0.9,1.5) ;$ similarly,

$$
\begin{aligned}
& M\left(\dot{S}_{2}\right)=(0.8,1.1,1.0), \\
& M\left(\dot{S}_{3}\right)=(0.9,1.2,1.0) .
\end{aligned}
$$

The strong independent number of $G$ is $D_{0}=\left(D_{10}, D_{20}, D_{30}\right)=(0.9,1.2,1.0)$. The maximum SIS is $\dot{S}$ with $M\left(\dot{S}_{3}\right)=D_{0}$.

3 The SECs are $T_{1}=\{(a, \beta),(c, d)\}$ and $\dot{T}_{2}=\{(a, \beta),(a$, $c),(c, d)\}$.

The value of SEC is calculated by

$$
\begin{aligned}
M(\dot{T}) & =\left(M_{1}(\dot{T}), M_{2}(\dot{T}), M_{3}(\dot{T})\right) \\
& =\left(\sum_{(u, v) \in \dot{T}} m_{2}(u, v), \sum_{(u, v) \in \dot{T}} \alpha_{2}(u, v), \sum_{(u, v) \in \dot{T}} n_{2}(u, v)\right) .
\end{aligned}
$$

Consequently,

$M\left(\dot{T}_{1}\right)=(0.6+0.3,0.7+0.5,0.2+0.8)=(0.9,1.2,1.0)$ and $M\left(\dot{T}_{2}\right)=(1.1,1.6,1.7)$ are the values of SECs $\dot{T}_{1}$ and $\dot{T}_{2}$, respectively. So, the SEC number is $C_{1}=\left(C_{11}, C, C_{31}\right)=$ $(0.9,1.2,1.7)$. None of the strong covers is a minimum SEC.

The only SM of $G$ is $\widetilde{O}=\{(a, \beta),(c, d)\}$. The SM number is defined by

$$
\begin{aligned}
M(\ddot{\tilde{O}}) & =\left(M_{1}(\ddot{\widetilde{O}}), M_{2}(\ddot{\tilde{O}}), M_{3}(\ddot{\tilde{O}})\right) \\
& =\left(\sum_{(u, v) \in \tilde{\tilde{O}}} m(u, v), \sum_{(u, v) \in \dot{\tilde{O}}} \alpha_{2}(u, v), \sum_{(u, v) \in \dot{\tilde{O}}} n_{2}(u, v)\right) .
\end{aligned}
$$

Thus,

$M(\ddot{\widetilde{O}})=(0.6+0.3,0.7+0.5,0.2+0.8)=(0.9,1.2,1.0) \quad$ is the SM number.

Theorem 13. Let $G=(V, E)$ be an $S V N G$ of order $(m, n)$ which does not contain any isolated vertices; then, for every such graph,

(i) $C_{0}+D_{0}=M(V) \leq m$

(ii) $C_{1}+D_{1} \geq n$

Proof. Let $\dot{Y}_{0}$ be the minimum SVC of $G$ and $C_{0}=M\left(\dot{Y}_{0}\right)$. Then $V-\dot{Y}_{0}$ is an SIS of vertices. That means $V-\dot{Y}_{0}$ contains such vertices that are incident on SEs of $G$. So, $D_{0} \geq M\left(V-\dot{Y}_{0}\right)=M(V)-C_{0}$.

$$
\text { i.e. } C_{0}+D_{0} \geq M(V) \text {. }
$$

Let $\widehat{Z}_{0}$ be the maximum SIS of vertices in $G$ and $D_{0}=M\left(\hat{Z}_{0}\right)$. It means $\widehat{Z}_{0}$ does not contain any vertices that are connected to each other via SE and therefore all the SEs of $G$ are strongly covered by the vertex in $V-\widehat{Z}_{0}$. Hence, $V-\widehat{Z}_{0}$ is an SVC of $G$ with smallest value $C_{0}$. Therefore, $C_{0} \leq M\left(V-\widehat{Z}_{0}\right)=M(V)-D_{0}$.

$$
\text { i.e. } C+D_{0} \leq M(V) \text {. }
$$

Using (46) and (47),

$$
C_{0}+D_{0}=M(V) .
$$

By definition, $M(V)$ is as follows:

$$
M(V) \leq m .
$$

Hence,

$$
C_{0}+D_{0}=M(V) \leq m
$$

As $C_{1}$ and $D_{1}$ are determined by considering the value of the SEs and the sum of the vertex values is represented by $m$, the second inequality follows directly.

Definition 14. Suppose that $\ddot{\widetilde{O}}$ is an SM in an SVNG G= $(V, E)$ and if $\tilde{\widetilde{O}}$ strongly matches all vertices of $G$ to some vertices of $G$, then $\ddot{\widetilde{O}}$ is said to be perfect strong matching (PSM).

Example 5. All of the edges of an SVNG $G=(V, E)$ are strong and are represented by solid lines, as shown in Figure 5. Therefore, the ..SMs are the collection of $\ddot{\widetilde{O}}_{1}=\{(a, \beta),(c, d)\}, \quad \ddot{\widetilde{O}}_{2}=\{(a, c),(\beta, d)\}, \quad$ and $\widetilde{O}_{3}=\{(a, d), .(\beta, c)\}$. Moreover, each of the collections $\widetilde{O}_{1}, \tilde{O}_{2}$, and $\widetilde{O}_{3}$ is also a PSM. The SM number is calculated by

$$
\begin{aligned}
M(\ddot{\tilde{O}}) & =\left(M_{1}(\ddot{\tilde{O}}), M_{2}(\ddot{\widetilde{O}}), M_{3}(\ddot{\tilde{O}})\right) \\
& =\left(\sum_{(u, v) \in \ddot{\tilde{O}}} m_{2}(u, v), \sum_{(u, v) \in \tilde{\tilde{O}}} \alpha_{2}(u, v), \sum_{(u, v) \in \ddot{\tilde{O}}} n_{2}(u, v)\right) .
\end{aligned}
$$




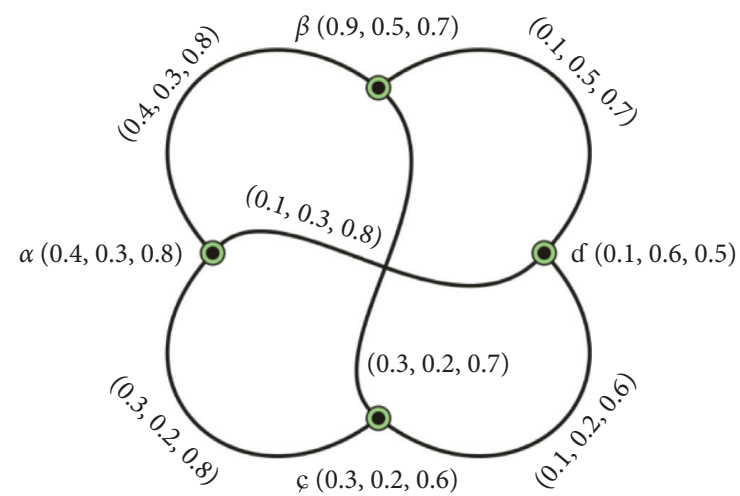

FIGURE 5: Perfect strong matching in SVNG.

Hence, the SM numbers are

$M\left(\ddot{\widetilde{O}}_{1}\right)=(0.4+0.3,0.3+0.2,0.8+0.6)=(0.7,0.5,1.4)$.

Similarly, $\quad M\left(\ddot{\widetilde{O}}_{2}\right)=(0.4,0.7,1.5) \quad$ and $\quad M\left(\ddot{\widetilde{O}}_{3}\right)=$ $(0.4,0.5,1.5)$.

\section{Paired Domination in Single-Valued Neutrosophic Graph}

This section introduces the concept of paired domination (PD), strong paired domination (SPD), and perfect paired domination. A couple of theorems are proved, and examples are also given to explain the concepts.

Definition 15. Suppose that $G$ denotes an SVNG and $v$ is a vertex in $G$; then, each of the neighbors of $v$ and $v$ itself is strongly dominated by $v$, which means that the vertices in $\dot{Y}[v]$ are strongly dominated by $v$. If every vertex of $V(G)-\dot{S}$ is a strong neighbor of some vertex in $\dot{S}$ of vertices of $G$, then $\dot{S}$ is a strong dominating collection of $G$.

Definition 16. $M_{1}(\dot{S})=\sum_{u \in \dot{S}} m_{2}(u, v)$ is the membership value, $M_{2}(\dot{S})=\sum_{u \in \dot{S}} \alpha_{2}(u, v)$ is the abstinence value, and $M_{3}(\dot{S})=\sum_{u \in \dot{S}} n_{2}(u, v)$ is the nonmembership value of the strong dominating collection $\dot{S}$, where $m_{2}(u, v)$ and $\alpha_{2}(u, v)$ represent the minimum of membership values and minimum of abstinence values of the SEs incident on $u$, while $n_{2}(u, v)$ represents the maximum of the values of nonmembership of such edges.

$B(G)=B=\left(B_{1}, B_{2}, B_{3}\right)$ denotes and defines the strong domination number of an SVNG $G$, where $B_{1}$ and $B_{2}$ represent the minimum membership value and minimum abstinence value of strong dominating collections of $G$, while $B_{3}$ represents the maximum nonmembership value of such collections.

Definition 17. If a collection $\dot{S} \subseteq V$ of vertices of an SVNG $G=(V, E)$ such that $\dot{S}$ is a strong dominating collection and the induced single-valued neutrosophic subgraph (SVNSG) $\dot{S}$ has a perfect strong matching, then $\dot{S}$ is called a strong paired dominating collection. $M_{1}(\dot{S})=\sum_{u \in \dot{S}} m_{2}(u, v)$ and
$M_{2}(\dot{S})=\sum_{u \in \dot{S}} \alpha_{2}(u, v)$ are the membership value and abstinence value of the strong paired dominating collection $\dot{S}$, while $M_{3}(\dot{S})=\sum_{u \in \dot{S}} n_{2}(u, v)$ is the nonmembership value of $\dot{S}$, where $m_{2}(u, v)$ and $\alpha_{2}(u, v)$ represent the minimum values of the membership and minimum values of the abstinence of SEs incident on $u$, while $n_{2}(u, v)$ represents the maximum values of the nonmembership of such edges.

$\delta(G)=\delta=\left(\delta_{1}, \delta_{2}, \delta_{3}\right)$ denotes and defines the SPD number of an SVNG $G$, where $\delta_{1}$ and $\delta_{2}$ represent the minimum membership value and minimum abstinence value of strong paired dominating collections of $G$, while $\delta_{3}$ represents the greatest nonmembership value of such sets.

Example 6. Figure 6 displays an SVNG $G=(V, E)$. In $G$, the edges represented by solid lines $(a, \beta),(a, c)$, and $(c, d)$ are the SEs, while $(\beta, d)$ is not a SE, which is represented by a dotted line. So, the strong dominating collections are $\dot{S}_{1}=\{a, \beta\}, \dot{S}_{2}=\{c, d\}$, and $\dot{S}_{3}=\{a, \beta, c, d\}$.

Now, the values of strong dominating collections are to be found by

$$
\begin{aligned}
M(\dot{S}) & =\left(M_{1}(\dot{S}), M_{2}(\dot{S}), M_{3}(\dot{S})\right) \\
& =\left(\sum_{u \in \dot{S}} m_{2}(u, v), \sum_{u \in \dot{S}} \alpha_{2}(u, v), \sum_{u \in \dot{S}} n_{2}(u, v)\right) .
\end{aligned}
$$

Therefore,

$M\left(\dot{S}_{1}\right)=(0.2+0.6,0.4+0.7,0.7+0.2)=(0.8,1.1,0.9)$.

Similarly, $\quad M\left(\dot{S}_{2}\right)=(0.5,0.9,1.6) \quad$ and $M\left(\dot{S}_{3}\right)=(1.3,2.0,2.5)$.

The SPD number is defined as

$$
\delta(G)=\left(\delta_{1}, \delta_{2}, \delta_{3}\right)
$$

Thus, $\delta(G)=(0.5,0.9,2.5)$ is the SPD number of SVNG in Figure 6.

Theorem 14. If $G=(V, E)$ is a complete SVNG, then $\delta_{1}(G)=2 m_{2}(u, v), \quad \delta_{2}(G)=2 \alpha_{2}(u, v), \quad$ and $\delta_{3}(G)=2 n_{2}(u, v)$, where $m_{2}(u, v), \alpha_{2}(u, v)$, and $n_{2}(u, v)$ are the membership, abstinence, and nonmembership values of the weakest edge in $G$.

Proof. Since, by definition of a complete SVNG G, each vertex of $G$ is connected to every other vertex in $G$ and all of its edges are strong, let $\{u, v\}$ be two vertices in any collection of $G$, then a strong paired dominating collection is formed by such set. Thus, $\delta_{1}(G)=m_{2}(u, v)+m_{2}(u, v)=2 m_{2}(u, v)$, $\delta_{2}(G)=\alpha_{2}(u, v)+\alpha_{2}(u, v)=2 \alpha_{2}(u, v), \quad$ and $\delta_{3}(G)=n_{2}(u, v)+n_{2}(u, v)=2 n_{2}(u, v)$.

Theorem 15. The SPD numbers for a complete bipartite SVNG $K$ are $\delta_{1}(K)=2 m_{2}(u, v), \delta_{2}(K)=2 \alpha_{2}(u, v)$, and $\delta_{3}(K)=2 n_{2}(u, v)$, where $m_{2}(u, v), \alpha_{2}(u, v)$, and $n_{2}(u, v)$ are the membership, abstinence, and nonmembership values of the weakest edge in $K$.

Proof. By definition of $K$, all of its edges are strong. Moreover, each of the vertices in $V_{1}$ is connected to all the 


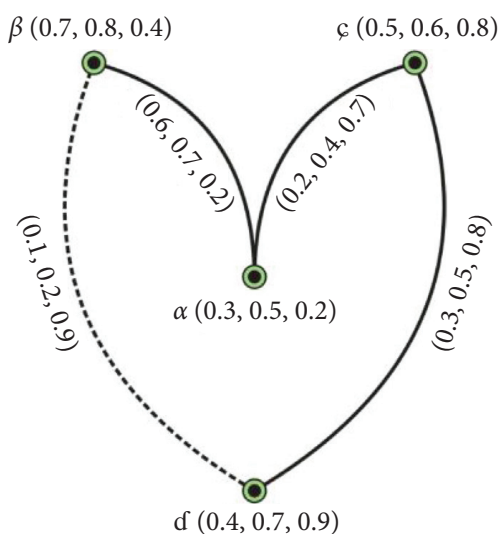

Figure 6: Paired domination in SVNG.

vertices in $V_{2}$. Therefore, any collection in $K$ which contains two vertices, one in $V_{1}$ and the other in $V_{2}$, is a strong paired dominating collection. Let $\{u, v\}$ be the end vertices of any weakest edge $(u, v)$ in $K$ such that $u \in V_{1}$ and $v \in V_{2}$; then $\{u, v\}$ makes a strong paired dominating collection.

Thus, $\delta_{1}(K)=m_{2}(u, v)+m_{2}(u, v)=2 m_{2}(u, v), \delta_{2}(K)=$ $\alpha_{2}(u, v) \quad+\alpha_{2}(u, v)=2 \alpha_{2}(u, v), \quad$ and $\quad \delta_{3}(K)=n_{2}(u$, $v)+n_{2}(u, v)=2 n_{2}(u, v)$.

\section{Conclusion}

Single-valued neutrosophic sets are characterized by three independent fuzzy-valued mappings, that is, membership grade, abstinence grade, and nonmembership grade. This research presented the notions of covering, matching, and paired domination using the strong edges in the neutrosophic environment. Furthermore, this paper discussed the concepts of strong vertices, strong edges, strong vertex cover, strong edge cover, strong vertex covering number, strong edge covering number, strong independent number, strong matching, and strong paired domination number in SVNG by strong edges with suitable examples. Moreover, the strong independent number, the strong matching, and the strong paired domination number of complete SVNG and complete bipartite are determined. The relations among these notions and numerous interesting properties are also proposed. Many interesting results of covering, matching, and paired domination are obtained and proved for SVNGs, complete SVNGs, SVNC, and complete bipartite SVNGs.

\section{Data Availability}

The data used to support the findings of this study are included within the article.

\section{Conflicts of Interest}

The authors declare that there are no conflicts of interest regarding the publication of this article.

\section{Acknowledgments}

This work was supported by the Major Project of Education Department in Hebei (No. ZD2021039) and Innovation and Improvement Project of Academic Team of Hebei University of Architecture (Mathematics and Applied Mathematics) (No. TD202006).

\section{References}

[1] F. Smarandache, "A unifying field in logics: neutrosophic logic," Neutrosophy, Neutrosophic Set, Neutrosophic Probability. Infinite Study, American Research Press Rehoboth, McKinley County, NM, USA, 2005.

[2] L. A. Zadeh, "Fuzzy sets," Information and Control, vol. 8, no. 3, pp. 338-353, 1965.

[3] K. T. Atanassov, "Intuitionistic fuzzy sets," Fuzzy Sets and Systems, vol. 20, no. 1, pp. 87-96, 1986.

[4] K. T. Atanassov, "Intuitionistic fuzzy sets," in Proceedings of the VII ITKR's Session, Sofia, Bulgarian, June 1983.

[5] K. T. Atanassov, "Intuitionistic fuzzy sets," in Intuitionistic Fuzzy Sets, pp. 1-137, Springer, Berlin, Germany, 1999.

[6] F. Smarandache, "Neutrosophic set-a generalization of the intuitionistic fuzzy set," International Journal of Pure and Applied Mathematics, vol. 24, no. 3, p. 287, 2005.

[7] H. Wang, F. Smarandache, Y. Zhang, and R. Sunderraman, "Single valued neutrosophic sets," Multispace and Multistructure, vol. 4, 2010.

[8] A. Rosenfeld, "Fuzzy graphs," in Fuzzy Sets and Their Applications to Cognitive and Decision Processes, pp. 77-95, Elsevier, Amsterdam, Netherlands, 1975.

[9] A. Kaufmann, Introduction to the Theory of Fuzzy Subsets, Vol. 2, Academic Press, Cambridge, MA, USA, 1975.

[10] A. N. Gani and K. Radha, "On regular fuzzy graphs," Journal of Physical Science, vol. 12, pp. 33-40, 2008.

[11] S. Samanta and M. Pal, "Fuzzy tolerance graphs," International Journal of Latest Trends in Mathematics, vol. 1, no. 2, pp. 57-67, 2011.

[12] H. Rashmanlou, S. Samanta, S. M. Pal, and R. A. Borzooei, "A study on bipolar fuzzy graphs," Journal of Intelligent \& Fuzzy Systems, vol. 28, no. 2, pp. 571-580, 2015.

[13] H. Rashmanlou, S. Samanta, M. Pal, and R. A. Borzooei, "Bipolar fuzzy graphs with categorical properties," International Journal of Computational Intelligence Systems, vol. 8, no. 5, pp. 808-818, 2015.

[14] S. Samanta, M. Pal, and A. Pal, "Some more results on bipolar fuzzy sets and bipolar fuzzy intersection graphs," The Journal of Fuzzy Mathematics, vol. 22, no. 2, pp. 253-262, 2014.

[15] M. Pal, S. Samanta, and H. Rashmanlou, "Some results on interval-valued fuzzy graphs," International Journal of Computer Science and Electronics Engineering, vol. 3, no. 3, pp. 205-211, 2015.

[16] H. Rashmanlou and M. Pal, "Balanced interval-valued fuzzy graphs," Journal of Physical Sciences, vol. 17, pp. 43-57, 2013.

[17] S. Samanta, A. Pal, and M. Pal, "New concepts of fuzzy planar graphs," International Journal of Advanced Research in Artificial Intelligence, vol. 3, no. 1, pp. 52-59, 2014.

[18] S. Samanta and M. Pal, "Fuzzy planar graphs," IEEE Transactions on Fuzzy Systems, vol. 23, no. 6, pp. 1936-1942, 2015.

[19] S. Samanta and M. Pal, "Bipolar fuzzy hypergraphs," International Journal of Fuzzy Logic Systems, vol. 2, no. 1, pp. 17-28, 2012. 
[20] S. Samanta and M. Pal, "Irregular bipolar fuzzy graphs," International Journal of Applications of Fuzzy Sets, vol. 2, pp. 91-102, 2012.

[21] S. Samanta, B. Sarkar, D. Shin, and M. Pal, "Completeness and regularity of generalized fuzzy graphs," SpringerPlus, vol. 5, no. 1, pp. 1979-2003, 2016.

[22] A. Shannon and K. Atanassov, "On a generalization of intuitionistic fuzzy graphs," NIFS, vol. 12, pp. 24-29, 2006.

[23] M. Akram and B. Davvaz, "Strong intuitionistic fuzzy graphs," Filomat, vol. 26, no. 1, pp. 177-196, 2012.

[24] M. Akram and W. A. Dudek, "Intuitionistic fuzzy hypergraphs with applications," Information Sciences, vol. 218, pp. 182-193, 2013.

[25] M. G. Karunambigai, M. Akram, S. Sivasankar, and K. Palanivel, "Balanced intuitionistic fuzzy graphs," Applied Mathematical Sciences, vol. 7, pp. 2501-2514, 2013.

[26] S. Sahoo, M. Pal, H. Rashmanlou, and R. A. Borzooei, "Covering and paired domination in intuitionistic fuzzy graphs," Journal of Intelligent \& Fuzzy Systems, vol. 33, no. 6, pp. 4007-4015, 2017.

[27] S. Sahoo and M. Pal, "Intuitionistic fuzzy competition graphs," Journal of Applied Mathematics and Computing, vol. 52, no. 1-2, pp. 37-57, 2015.

[28] S. Sahoo and M. Pal, "Intuitionistic fuzzy tolerance graphs with application," Journal of Applied Mathematics and Computing, vol. 55, no. 1-2, p. 495, 2016.

[29] F. Smarandache, "Refined literal indeterminacy and the multiplication law of sub-indeterminacies," Neutrosophic Sets and Systems, vol. 9, pp. 58-2015.

[30] F. Smarandache, Types of Neutrosophic Graphs and neutrosophic Algebraic Structures together with their Applications in Technology, Seminar, Universitatea Transilvania din Brasov, Facultatea de Design de Produs si Mediu, Brasov, Romania, 2015.

[31] F. Smarandache, Symbolic Neutrosophic Theory, Europanova ASBL, Brussels, Belgium, 2015.

[32] G. Garg, K. Bhutani, M. Kumar, and S. Aggarwal, "Hybrid model for medical diagnosis using neutrosophic cognitive maps with genetic algorithms," in Proceedings of the IEEE International Conference on Fuzzy Systems (FUZZ-IEEE 2015), Istanbul, Turkey, August 2015.

[33] W. B. Vasantha Kandasamy, K. Ilanthenral, and F. Smarandache, Neutrosophic Graphs: A New Dimension to Graph Theory, Kindle Edition, 2015.

[34] J. Ye, "Single-valued neutrosophic minimum spanning tree and its clustering method," Journal of Intelligent Systems, vol. 23, no. 3, pp. 311-324, 2014.

[35] H.-L. Yang, Z.-L. Guo, Y. She, and X. Liao, "On single valued neutrosophic relations," Journal of Intelligent \& Fuzzy Systems, vol. 30, no. 2, pp. 1045-1056, 2016.

[36] S. Naz, H. Rashmanlou, and M. A. Malik, "Operations on single valued neutrosophic graphs with application," Journal of Intelligent \& Fuzzy Systems, vol. 32, no. 3, pp. 2137-2151, 2017.

[37] M. Akram, N. Waseem, and W. A. Dudek, "Certain types of edge m-polar fuzzy graphs," Iranian Journal of Fuzzy Systems, vol. 14, pp. 27-50, 2017.

[38] M. Akram, "Certain bipolar neutrosophic competition graphs," The Journal of the Indonesian Mathematical Society, vol. 24, pp. 1-25, 2017.

[39] M. Akram and S. Siddique, "Neutrosophic competition graphs with applications," Journal of Intelligent \& Fuzzy Systems, vol. 33, no. 2, pp. 921-935, 2017.
[40] M. Akrama, Single-Valued Neutrosophic Planar Graphs; Infinite Study, Modern Science Publisher, New York, NY, USA, 2016.

[41] H. Garg, "Decision making analysis based on sine trigonometric operational laws for single-valued neutrosophic sets and their applications," Applied and Computational Mathematics, vol. 19, no. 2, pp. 255-276, 2020.

[42] H. Garg, "Novel neutrality aggregation operator-based multiattribute group decision-making method for single-valued neutrosophic numbers," Soft Computing, vol. 24, no. 14, pp. 10327-10349, 2020.

[43] H. Garg, "Multiple attribute decision making based on immediate probabilities aggregation operators for single-valued and interval neutrosophic sets," Journal of Applied Mathematics and Computing, vol. 63, no. 1-2, pp. 619-653, 2020.

[44] S. Broumi, M. Talea, A. Bakali, and F. Smarandache, "Single valued neutrosophic graphs," Journal of New Theory, vol. 10, pp. 86-101, 2016.

[45] S. Broumi, F. Smarandache, M. Talea, and A. Bakali, "An introduction to bipolar single valued neutrosophic graph theory," in Applied Mechanics and Materials, vol. 841, pp. 184-191, Trans Tech Publications Ltd, Freienbach, Switzerland, 2016.

[46] S. Broumi, F. Smarandache, M. Talea, and A. Bakali, "Single valued neutrosophic graphs: degree, order and size," in Proceedings of the 2016 IEEE International Conference on Fuzzy Systems (FUZZ-IEEE), pp. 2444-2451, IEEE, Istanbul, Turkey, July 2016.

[47] M. Akram and G. Shahzadi, Operations on Single-Valued Neutrosophic Graphs, Infinite Study, Springer, Berlin, Germany, 2017.

[48] F. Karaaslan and B. Davvaz, "Properties of single-valued neutrosophic graphs," Journal of Intelligent \& Fuzzy Systems, vol. 34, no. 1, pp. 57-79, 2018.

[49] G. Ghorai and M. Pal, "Ceratin types of product bipolar fuzzy graphs," International Journal of Applied and Computational Mathematics, vol. 3, no. 2, pp. 605-619, 2015. 